HNO 2022 $\cdot 70: 418-421$

https://doi.org/10.1007/s00106-022-01151-3

Angenommen: 21. Oktober 2021

Online publiziert: 4. März 2022

(c) Der/die Autor(en) 2022

\section{Geschichtliche Entwicklung der rekonstruktiven Chirurgie in der Onkologie des HNO-Bereichs}

Friedrich Bootz

Univ.-HNO-Klinik, Bonn, Deutschland

Die rekonstruktive Chirurgie ist ein wichtiger Bestandteil der Tumorchirurgie des Kopf-Hals-Bereichs. Große ablative Tumorresektionen wurden bereits Anfang des 20. Jahrhunderts durchgeführt, nachdem es möglich wurde, durch verbesserte Operationstechnik den intraoperativen Blutverlust zu reduzieren bzw. durch Transfusionen auszugleichen. Ein weiterer Meilenstein war die postoperative Infektprophylaxe durch die Einführung der Antibiotika. John Conley erkannte als einer der Pioniere der radikalen Tumorchirurgie die dringende Notwendigkeit rekonstruktiver Maßnahmen. Die Anfänge waren jedoch begleitet von funktionellen postoperativen Einschränkungen, die schließlich erst durch die Einführung des Deltopektoral- und des Pektoralis-major-Lappens verbessert werden konnten. Ein weiterer Schritt war die Einführung der mikrovaskulären Transplantate, die eine bessere, der Situation angepasste Rekonstruktion erlaubte. Anfangs waren jedoch die Komplikationsraten aufgrund mangelnder Technik der Anastomosierung kleiner Gefäße und unzureichender Instrumente sehr hoch. Daher konnte sich diese Methode nur schleppend durchsetzen. Die Techniken der Lappenentnahme und der Mikrogefäßanastomose entwickelten sich jedoch weiter, sodass die mikrovaskuläre Gewebetransplantation zu einer zuverlässigen Methode wurde, die heute zum Standardrepertoire der rekonstruktiven Chirurgie gehört.

\title{
Schlüsselwörter
}

Kopf-Hals-Karzinome · Rekonstruktive Maßnahmen · Mikrovaskuläre Lappen · Regionale Lappen · Geschichte

Die Entwicklung der radikalen Tumorchirurgie, die von den Allgemeinchirurgen William Halsted konzipiert und von Hayes Martin umgesetzt wurde, war nur durch Fortschritte bei der Minimierung bis dahin häufig aufgetretener Komplikationen wie Infektionen und Blutverlust möglich. Durch die Verbesserung der Operationstechniken und der Weiterentwicklung des Instrumentariums konnten die Komplikationen reduziert werden. Zusätzlich spielte die Entdeckung der Antibiotika in den 1930erund 1940er-Jahren eine große Rolle. Dennoch waren komplexere Tumorresektionen durch das Fehlen entsprechender rekonstruktiver Maßnahmen eingeschränkt bzw. hatten erhebliche funktionelle Beeinträchtigungen zur Folge.
Auf dem Gebiet der ablativen Chirurgie von Kopf- und Halstumoren machte sich der HNO-Arzt John Conley aus New York bald einen Namen und erlangte internationalen Ruf. Er war ein früher Verfechter der rekonstruktiven Chirurgie. Bereits 1953 veröffentlichte er einen Artikel dazu [1]. Er propagierte ein einzeitiges Vorgehen der radikalen Tumorexzision mit sofortiger Rekonstruktion, wobei er zur Pharynxrekonstruktion Vollhauttransplantate einsetzte, die jedoch zu erheblicher Vernarbung und Stenosierung führten. Anfangs verwandte er ausschließlich lokale Hautlappen, wie z.B. den Stirnlappen, zur Rekonstruktion in der Mundhöhle bzw. im Oropharynx, die allerdings erhebliche Entstellungen zur Folge hatten. Über ein Jahrzehnt lang war eine Rekonstruktion nur mit denen von 
Conley beschriebenen lokalen Lappen und Hauttransplantaten möglich, bis der Deltopektorallappen von Bakamjian 1968 entwickelt und bekannt gemacht wurde [2, 3]. In seiner Publikation beschrieb er die Rekonstruktion des Pharynx mit einem medial basierten Deltopektorallappen. Aber auch der Deltopektorallappen hatte häufig erhebliche ästhetische Nachteile zur Folge und verlangte oft ein zweizeitiges Vorgehen, insbesondere bei der Rekonstruktion des Pharynx.

Ein weiterer Schritt in der rekonstruktiven Chirurgie war die Beschreibung des Pektoralis-major-Myokutanlappens durch Ariyan [4]. Er stellte in seiner Publikation die Lappenanatomie und die entsprechende Entnahmetechnik dar, die er erfolgreich bei vier Patienten zum Verschluss großer Defekte im Kopf-Hals-Bereich einsetzte. Diese Technik führte zu einer neuen Ära der Kopf- und Halsrekonstruktion, die es ermöglichte, viele komplexe Gewebsdefekte einzeitig mit einem Lappen mit guten funktionellen Ergebnissen durch den KopfHals-Chirurgen zu verschließen.

Mikrovaskuläre Techniken zur Übertragung von freiem Gewebe entwickelten sich aus der Gefäßchirurgie, wobei mehrere technische Probleme wie das Fehlen einer adäquaten Vergrößerung und die Prophylaxe von Thrombosen ihre frühe Verbreitung verhinderten.

Wesentlich für die Entwicklung der Mikrovaskularchirurgie war neben der Lupenbrille die Einführung des Operationsmikroskops, das in den 1950er-Jahren von Wullstein zusammen mit der Fa. Zeiss, Oberkochen, Deutschland als OPMI 1 entwickelt wurde.

Ein Bericht von Jacobson und Suarez [5] aus dem Jahr 1962 über die Langzeitdurchgängigkeit mikrovaskulärer Anastomosen bei Hunden und Kaninchen leitete eine neue Ära der mikrovaskulären Forschung ein. Bereits 1950 hatte Bernard Seidenberg, ein Gefäßchirurg, 300 Fälle mikrovaskulärer Dünndarmtransplantationen bei Hunden vorgenommen und deren Ergebnisse veröffentlicht [6], bevor er die erste Autotransplantation bei einem Menschen vornahm, der leider den Eingriff nicht überlebte. Im Jahr 1961 beschrieben Roberts und Douglass dann den ersten erfolgreichen Ersatz des zervikalen Ösophagus und des Hypopharynx beim Menschen mithil- fe eines revaskularisierten freien Jejunumsegments [7].

Die ersten mikrovaskulären Transplantate in der Kopf-Hals-Chirurgie wurden dann in den 1970er-Jahren an mehreren Institutionen klinisch eingesetzt, wobei der Erfolg lediglich bei etwa $70 \%$ lag. William Panje und Shan Baker veröffentlichten 1977 die erste Serie von Rekonstruktionen der Mundhöhle mit einem freien Leistenlappen [8]. Der mikrovaskuläre Gewebetransfer konnte sich jedoch wegen Problemen bei der Anastomosierung der kleinen Gefäße mit sehr häufig auftretenden Gefäßverschlüssen und dadurch bedingten erheblichen postoperativen Komplikationen nicht durchsetzen. Auf der Suche nach anderen rekonstruktiven Techniken kam man wieder auf die gestielten, insbesondere den Pektoralis-major-Lappen zurück, die sich als sehr zuverlässig erwiesen und die weitere Entwicklung des mikrovaskulären Gewebetransfers behinderten. Bald entwickelten sich der Pektoralis-major- und der Deltopektorallappen zu den Standardinstrumenten der rekonstruktiven Chirurgie des Kopf-Hals-Bereichs. Auch Conley sprach sich für die Überlegenheit der gestielten Lappen gegenüber dem freien Gewebetransfer aus und hat diese noch in der zweiten Auflage seines Standardwerks, das im Jahr 1989 erschien, ausführlich dargestellt.

Man erkannte jedoch bald die Grenzen der gestielten Transplantate. Nicht alle Regionen des Kopf-Hals-Bereiches konnten damit erreicht werden, und der GefäßMuskel-Stiel war oft ein Hindernis. Häufig war ein zweizeitiges Vorgehen notwendig. Ferner waren die funktionellen und ästhetischen Ergebnisse meist nicht zufriedenstellend.

Die besonderen Erfordernisse der Rekonstruktion nach ausgedehnten Tumorresektionen und die höheren Ansprüche an die funktionellen und ästhetischen Ergebnisse sorgten dafür, dass die Techniken des freien Gewebetransfers wieder aufgegriffen und weiterentwickelt wurden.

Die exakten anatomischen Studien durch McGregor im Jahr 1973 über Gefäßverläufe, Gefäßdurchmesser und Versorgungsgebiete der zuführenden Gefäße verschiedener Körperregionen und der Definition der „axial pattern flaps“ stellten die Basis zur weiteren Entwicklung unterschiedlicher Transplantate dar [9]. Der revaskularisierte Latissimus-dorsiLappen wurde bereits Ende der 1970erJahre von Olivari beschrieben [10, 11]. 1979 beschrieb Taylor den revaskularisierten Beckenkamm an der A. circumflexa ileum profunda [12], der sich zu einem Standardtransplantat zur Rekonstruktion des Unterkiefers entwickelte und heute noch in dieser Form eingesetzt wird. Die Fibula war jedoch der erste Knochen, der mit Erfolg als vaskularisiertes Transplantat eingesetzt wurde, und zwar zur Überbrückung ausgedehnter Langknochendefekte, wie von Taylor bereits 1975 berichtet [13]. Zur Rekonstruktion des Unterkiefers wurde dieses Transplantat allerdings zum ersten Mal 1989 von Hidalgo eingesetzt [14]. Heute stellt die Fibula das Standardtransplantat zur Rekonstruktion des Unterkiefers dar.

Die Bedeutung des Rektus-abdominisLappens, der sich v. a. in den USA zum „Arbeitspferd" der rekonstruktiven Chirurgie entwickelte, wurde von Brown et al. bereits 1975 erkannt [15]. 1980 wurde von Dos Santos der Skapulalappen [16] und 1982 von Nassif der Paraskapulalappen [17] beschrieben, beide an der A. circumflexa scapulae.

1978 berichteten Yang et al. [18] über den fasziokutanen Unterarmlappen, der 1982 von Mühlbauer et al. [19] außerhalb Chinas bekannt gemacht wurde. Er fand jedoch anfangs durch die hohen Komplikationsraten an der Entnahmeregion und durch den hauptsächlich von Kieferchirurgen vorgenommenen Einsatz des antimesenterisch aufgeschnittenen Jejunumsegments als Patch keine weite Verbreitung bei der Rekonstruktion in der Mundhöhle und im Pharynx. Die ersten klinischen Anwendungen des Jejunumpatchs wurden im Jahr 1971 von Black, Bevin und Arnold vorgenommen [20]. Die Technik geht auf Green und Som zurück, die erstmals 1966 solche Transplantationsversuche an Hunden durchführten [21]. Das Jejunumpatch wurde aufgrund seiner Schleimhautähnlichkeit und der ausgesprochen guten Modellierbarkeit favorisiert und anderen Transplantaten vorgezogen.

Soutar berichtete im Jahr 1986 über eine große Zahl von Rekonstruktionen nach Tumorresektion im Oropharynx und in der Mundhöhle mithilfe des Unterarmlappens 
[22]. In England hatte sich diese Methode an einigen wenigen Zentren zur Standardrekonstruktion entwickelt. Der Autor hatte bereits 1987 bei Philip Stell in Liverpool während eines einjährigen Aufenthalts die Gelegenheit, diese Technik zu erlernen und sie danach in Deutschland im Kopf-Hals-Bereich einzuführen. Allerdings war es anfangs aufgrund der als sehr hoch angesehenen Entnahmemorbidität $[23,24]$ schwierig, die Kopf-Hals-Chirurgen, insbesondere die Kieferchirurgen, von der Qualität des Transplantats zu überzeugen. Die klinische Erfahrung hat jedoch gezeigt, dass das Jejunumpatch mechanisch wenig belastbar ist und zu nicht unerheblicher Schrumpfung insbesondere nach Bestrahlung neigt. Häufig traten v.a. in mechanisch belasteten Regionen der Mundhöhle Ulzerationen, Blutungen und Nekrosen auf. Diese negative Eigenschaft des Jejunumtransplantats führte zu einem Aufschwung der fasziokutanen Lappen, insbesondere des Unterarmlappens, der mechanisch gut belastbar ist, nur eine geringe Schrumpfungsneigung zeigt und fast ebenso gut modellierbar ist wie das Jejunumsegment, allerdings keine schleimhautähnliche Eigenschaft besitzt.

Durch eine verbesserte Operationstechnik bei der Entnahme des Unterarmlappens konnte zudem die Morbidität deutlich gesenkt werden [25]. 1991 konnten wir unsere Erfahrungen in einem Buch [26] veröffentlichen und als Koautor einen MKG-Chirurgen gewinnen. Von dem Zeitpunkt an war das Unterarmtransplantat auch von MKG-Chirurgen akzeptiert und löste im Laufe der Zeit das Jejunumpatch zur Rekonstruktion im Bereich der Mundhöhle und des Pharynx ab.

Zur Rekonstruktion des Hypopharynx nach Pharyngolaryngektomie wurde über längere Zeit hinweg noch das Jejunum als Rohr eingesetzt. Doch eine nicht unerhebliche Anzahl von Komplikationen sowohl in der Empfängerregion als auch an der Entnahmestelle hat auch in diesem Bereich dazu geführt, dass nach anderen Methoden gesucht wurde. Harii beschrieb 1985 als Erster die Rekonstruktion des Hypopharynx mithilfe eines zum Rohr geformten Unterarmlappens [27]. Unsere Arbeitsgruppe hat daran eine Modifikation vorgenommen. Der Unterarmlappen wird nicht mehr zum Rohr geformt, sondern U-för- mig an die prävertebrale Faszie angeheftet [28].

Die Mikrogefäßanastomose hat seit ihrer Erstbeschreibung durch Carell, abgesehen von der Verbesserung des Nahtmaterials, des Instrumentariums, der Gefäßclips und der Vergrößerungsmöglichkeiten, keine wesentliche Entwicklung durchgemacht. Bereits Seidenberg verwandte 1958 eine Art von Konnektorsystem. Konnektoren wurden zwar weiterentwickelt, konnten jedoch die Mikrogefäßanastomose mit Nahttechnik bis heute nicht vollständig ersetzen.

Die Entwicklung des mikrovaskulären Gewebetransfers hat zu einer enormen Bereicherung der rekonstruktiven Chirurgie nach Tumorresektion im Kopf-Hals-Bereich beigetragen und gehört heute zum Standardrepertoire der Kopf-Hals-Onkologie. Es gibt eine hohe, fast unüberschaubare Vielfalt an freien Transplantaten, die nicht alle für die rekonstruktive Chirurgie des Kopf-Hals-Bereichs geeignet erscheinen. Der verantwortungsbewusste Operateur wird sich ein Repertoire an mikrovaskulären Lappen zulegen, mit dem es ihm möglich ist, den rekonstruktiven Anforderungen seines Fachgebiets gerecht zu werden. Neben mikrovaskulären Transplantaten, die aufgrund ihrer hohen Flexibilität sicherlich die erste Wahl sind, gibt es auch Situationen, in denen heute noch gestielte Lappen eingesetzt werden müssen, sodass sie nicht aus dem Repertoire der rekonstruktiven Techniken gestrichen werden dürfen.

Prälaminierten Lappen, bei denen z. B. auf Muskel- oder entepithelisierten fasziokutanen Lappen spezifisches Gewebe aufgebracht wird, können ebenfalls zur situationsgerechten Rekonstruktion eingesetzt werden. Solche Techniken werden seit mehreren Jahren beschrieben, wobei oft Schleimhaut aufgebracht wird [29, 30]. Zukünftige Entwicklungen können in Verfahren liegen, bei denen durch „tissue engineering" bedarfsgerechte Transplantate mit z.B. Schleimhaut, Knochen oder Knorpel geschaffen werden, wobei jedoch die bisherigen Ergebnisse eher ernüchternd sind. Ein Nachteil dieser Techniken ist darin zu sehen, dass eine einzeitige Rekonstruktion nach Tumorresektion nicht möglich ist. Diese neuen Ansätze zeigen die vielfältigen weiteren Möglichkeiten mikrovasku- lärer Transplantate in der rekonstruktiven Chirurgie der Onkologie des Kopf-Hals-Bereichs.

\section{Korrespondenzadresse}

\section{Prof. Dr. Dr. h.c. Friedrich Bootz}

Univ.-HNO-Klinik

Venusberg Campus 1, 53127 Bonn,

Deutschland

friedrich.bootz@ukbonn.de

Prof. Dr. Dr. h.c. Friedrich Bootz Em. Direktor der Univ.HNO-Klinik

Funding. Open Access funding enabled and organized by Projekt DEAL.

\section{Einhaltung ethischer Richtlinien}

Interessenkonflikt. F. Bootz gibt an, dass kein Interessenkonflikt besteht.

Für diesen Beitrag wurden von den Autoren keine Studien an Menschen oder Tieren durchgeführt. Für die aufgeführten Studien gelten die jeweils dort angegebenen ethischen Richtlinien.

Open Access. Dieser Artikel wird unter der Creative Commons Namensnennung 4.0 International Lizenz veröffentlicht, welche die Nutzung, Vervielfältigung, Bearbeitung, Verbreitung und Wiedergabe in jeglichem Medium und Format erlaubt, sofern Sie den/die ursprünglichen Autor(en) und die Quelle ordnungsgemäß nennen, einen Link zur Creative Commons Lizenz beifügen und angeben, ob Änderungen vorgenommen wurden.

Die in diesem Artikel enthaltenen Bilder und sonstiges Drittmaterial unterliegen ebenfalls der genannten Creative Commons Lizenz, sofern sich aus der Abbildungslegende nichts anderes ergibt. Sofern das betreffende Material nicht unter der genannten Creative Commons Lizenz steht und die betreffende Handlung nicht nach gesetzlichen Vorschriften erlaubt ist, ist für die oben aufgeführten Weiterverwendungen des Materials die Einwilligung des jeweiligen Rechteinhabers einzuholen.

Weitere Details zur Lizenz entnehmen Sie bitte der Lizenzinformation auf http://creativecommons.org/ licenses/by/4.0/deed.de.

\section{Literatur}

1. Conley JJ (1953) One-stage radical resection of cervical esophagus, larynx, pharynx, and neck, with immediate reconstruction. AMA Arch Otolaryngol 58:645-654

2. Bakamjian VY (1968) Total reconstruction of pharynx with medially based deltopectoral skin flap. NYState J Med 68:2771-2778

3. Bakamjian VY (1965) A two-stage method for pharyngoesophageal reconstruction with a primary pectoral skin flap. Plast Reconstr Surg 36:173-184 
4. Ariyan S (1979) The pectoralis major myocutaneous flap. A versatile flap for reconstruction in the head and neck. Plast Reconstr Surg 63:73-81

5. Jacobson JH 2nd, Suarez EL (1962) Microvascular surgery. Dis Chest 41:220-224

6. Seidenberg B, Rosenak SS, Hurwitt ES, Som ML (1959) Immediate reconstruction of the cervical esophagus by a revascularized isolated jejunal segment. Ann Surg 149:162-171

7. Roberts RE, Douglass FM (1961) Replacement of the cervical esophagus and hypopharynx by a revascularized free jejunal autograft. Report of a case successfully treated. N Engl J Med 264:342-344

8. Panje WR, Krause CJ, Bardach J, Baker SR (1977) Reconstruction of intraoral defects with the free groin flap. Arch Otolaryngol 103:78-83

9. McGregor IA, Morgan G (1973) Axial and random pattern flaps. Br JPlast Surg 26:202-213

10. Olivari N (1979) Use of thirty latissimus dorsi flaps. Plast Reconstr Surg 64:654-661

11. Olivari N (1976) The latissimus flap. Br J Plast Surg 29:126-128

12. Taylor GI, Townsend P, Corlett R (1979) Superiority of the deep circumflex iliac vessels as the supply for free groin flaps. Clinical work. Plast Reconstr Surg 64:745-759

13. Taylor Gl, Miller GD, Ham FJ (1975) The free vascularized bone graft. A clinical extension of microvascular techniques. Plast Reconstr Surg 55:533-544

14. Hidalgo DA (1989) Fibula free flap: a new method of mandible reconstruction. Plast Reconstr Surg 84:71-79

15. Brown RG, VasconezLO, Jurkiewicz MJ (1975) Transverse abdominal flaps and the deep epigastric arcade. Plast Reconstr Surg 55:416-421

16. dos Santos LF (1984) The vascular anatomy and dissection of the free scapular flap. Plast Reconstr Surg 73:599-604

17. Nassif TM, Vidal L, Bovet JL, Baudet J (1982) The parascapular flap: a new cutaneous microsurgical free flap. Plast Reconstr Surg 69:591-600

18. Yang G, Chen B, Gao Y, Liu X, Li J, Jiang S, HeS (1981) Forearm free skin flap transplantation. Natl Med J Chin 61:139

19. Mühlbauer W, Herndel E, Stock W (1982) The forearm flap. Plast Reconstr Surg 70:336-342

20. Black PW, Bevin AG, Arnold PG (1971) One-stage palate reconstruction with a free neo-vascularized jejunal graft. Plast Reconstr Surg 47:316-320

21. Green GE, Som ML (1966) Free grafting and revascularization of intestine. I. Replacement of the cervical esophagus. Surgery 60:1012-1016

22. Soutar DS, McGregor IA (1986) The radial forearm flap in intraoral reconstruction: the experience of 60 consecutive cases. Plast Reconstr Surg 78:1-8

23. Timmons MJ, Missotten FE, Poole MD, Davies DM (1986) Complications of radial forearm flap donor sites. Br J Plast Surg 39:176-178

24. Boorman JG, Brown JA, Sykes PJ (1987) Morbidity in the forearm flap donor arm. Br J Plast Surg 40:207-212

25. Bootz F, Biesinger E (1991) Reduction of complication rate at radial forearm flap donor sites. ORL J Otorhinolaryngol Relat Spec 53:160-164

26. Bootz F, Müller GH (1992) Mikrovaskuläre Gewebetransplantation im Kopf-Hals-Bereich. Thieme, Stuttgart

27. Harii K, Ebihara S, Ono I, Saito H, Terui S, Takato T (1985) Pharyngoesophageal reconstruction using a fabricated forearm free flap. Plast Reconstr Surg 75:463-476

\section{Historical development of reconstructive surgery in head and neck oncology}

Reconstructive surgery is an important part of tumor surgery to the head and neck region. Large ablative tumor resections were already performed at the beginning of the 20th century, after it became possible to reduce intraoperative blood loss through improved surgical techniques or to compensate for it with transfusions. Another milestone was postoperative infection prophylaxis through the introduction of antibiotics. As one of the pioneers of radical tumor surgery, John Conley recognized the urgent need for reconstructive procedures. However, the beginnings were accompanied by postoperative functional impairments, which could only be improved with introduction of the deltopectoral and pectoralis major flaps. Another step was the introduction of microvascular grafts, which enabled better, situation-adapted reconstruction. Initially, however, the complication rates were rather high due to the inadequate technique of anastomosing small vessels as well as inadequate instruments. As a result, these methods were slow to gain acceptance. However, flap harvesting and microvascular anastomosis techniques continued to evolve, making microvascular tissue transplantation a reliable method that is now part of the standard repertoire of reconstructive surgery.

\section{Keywords}

Head and neck cancer - Reconstructive surgical procedures - Microvascular free flaps - Regional flaps · History

28. Bootz F, Weber A, Oeken J, Keiner S (2002) Reconstruktion des Hypopharynx nach Pharyngolaryngektomie mit U-förmigem Unterarmlappen. Laryngorhinootologie 81:17-21

29. Delaere PR, Hardillo J, Hermans R, Van Den Hof B (2001) Prefabrication of composite tissue for improved tracheal reconstruction. Ann Otol Rhinol Laryngol 110:849-860

30. Cheng OT, Tamaki A, Rezaee RP, Zender CA (2016) Laryngotracheal reconstruction with a prefabricated fasciocutaneous free flap for recurrent papillary thyroid carcinoma. Head Neck 38:E2512-E2514 